\title{
Anxiety and depression of high risk pregnant women hospitalized in two public hospital settings in Greece

\section{Abstract}

Objective: Many studies have examined the prevalence of anxiety and depression in low-risk pregnant women. However, very few studies have explored the prevalence of anxiety and depressive symptoms in high-risk pregnancy. The aim of this study was to investigate the prevalence of antenatal anxiety (AA) and antenatal depression (AD) in high-risk pregnant women hospitalized for a medical disorder.

Methods: The sample of this study consisted of 133 pregnant women with gestational age from 9 to 37 weeks. Anxiety was measured with State-Trait Anxiety Inventory (STAI) and depression was measured with Edinburgh Postnatal Depression Scale (EPDS). Descriptive statistics, such as means, standard deviations, and frequencies, were used.

Results: The means for STAl-state and -trait scores were 49.3 and 45.1, respectively. The mean score for EPDS was 12.5. Nine percent of the participants have answered that quite often or sometimes had the thought of harming themselves. Almost $50 \%$ of participants had depressive symptoms (EPDS $>11$ ) and the majority of participants had high anxiety level.

Conclusion: The prevalence of antenatal anxiety and depression identified in this study is of concern. Screening tools for detecting antenatal anxiety and depressive symptoms in high-risk pregnant is crucial.
K. Gourounti'
V. Karapanou ${ }^{1}$,
N. Karpathiotaki ${ }^{1}$,
G. Vaslamatzis ${ }^{2}$

1 Department of Midwifery, TEl of Athens, Athens, Greece

2 Department of Psychiatry, Medical School, University of Athens, Athens, Greece

\section{Contact information:}

\section{Kleanthi Gourounti .}

Agnoston Martiron 33-37,

Nea Smirni, Athens, Greece

Tel: 6937673814

झ" clairegourounti@yahoo.gr

Keywords

Anxiety, depression, high-risk pregnancy, hospitalization, prevalence

\section{Introduction}

Pregnancy and the transition to parenthood have been linked to an increase in anxiety symptoms, depression symptoms, worry and stress [1]. Women with depression during pregnancy present higher risk for 
miscarriage, preterm birth, low-birth-weight infants, small-for-gestational-age neonates and preeclampsia $[2,3]$. Depression and anxiety have been found to be exacerbated by uncertainty during high-risk pregnancies in women suffering from a medical disorder [4]. Therefore, a better understanding of the prevalence of anxiety and depression in highrisk pregnancies especially in population of women who experience hospitalization during pregnancy is essential. Although, numerous studies have examined the prevalence and risk factors of anxiety and depression in pregnancy, there are limited studies that have explored the prevalence of depression and even fewer studies that have explored the prevalence of anxiety in high risk pregnancy.

Studies in the United States, United Kingdom, France and Singapore have estimated the prevalence of depressive disorders in high-risk pregnancies $[4,5,6,7,8]$. Study by King et al. found that high risk pregnant women have high levels of depressive symptomatology than control women [4]. Studies by Aduard et al. [5], Brandon et al. [6], Denis et al. [7], Thiagayson et al. [8] revealed that the prevalence of depression in high risk pregnancy ranged from $18 \%$ to $58 \%$. Study by King et al. found significantly higher Spielberger state and trait anxiety scores in high-risk pregnant women than in control women4. The recent study by Thiagayson et al. was the only study that assessed the prevalence of anxiety disorders in the high-risk pregnant population [8]. This study found that $12.5 \%$ of the high-risk pregnant women had anxiety disorder and 5\% had comorbid depression and anxiety.

Therefore, it is essential to investigate the prevalence of anxiety and depression of the high-risk pregnant women in order to implement interventions to reduce adverse pregnancy outcomes.

The aim of this study was to assess the emotional state (anxiety and depression) of women with medical disorders of pregnancy and included women both with pre-existing medical disorders such as diabetes mellitus type 2, and those with pregnancy- induced disorders, e.g., pre-eclampsia, premature labour, or hyperemesis gravidarum.

\section{Materials and Methods}

\section{Sample and data collection process}

This study was carried out between February and June 2014 at two of the largest public hospitals in Athens, Greece to achieve a representative database. The questionnaire was administered to a sample of 150 pregnant women with a gestational age of between 9 and 37 weeks, who were hospitalized in the inpatient antenatal ward. High-risk pregnancies encompassed such conditions as one or more of the following: past adverse obstetric history, preexisting medical conditions, preeclampsia, intrauterine growth restriction, preterm premature/rupture of membranes, placenta previa, placenta abruption, hyperemesis, fetal anomaly, multiple pregnancy, gestational diabetes, threatened preterm labor, twin-twin transfusion syndrome or others such as preexisting hypertension and cervical incompetence. Finally, 133 women accepted to participate (response rate $89 \%$ ). The pregnant women were informed of the study aim and protocol, and once they voluntarily agreed to participate, they were given an envelope containing the questionnaires and an informed consent form. The completed questionnaires and the signed consent form were returned directly to the researcher (within two-three weeks).

\section{Study instruments}

State and trait anxiety was measured with the State Trait Anxiety Inventory (STAI) [9]. State anxiety is defined as an unpleasant emotional condition that emerges in case of threatening demands or dangers. Therefore, it should be low in non-stressful situations and high if circumstances are perceived to be threatening or dangerous. The state scale consists of 20 items that ask people to describe how they feel at a particular moment in time, ra- 
ted on a four- point scale ranging from not at all (1) to very much so (4). The trait scale consists of 20 items and ask people to describe how they generally feel (e.g., confident), rated on a four-point frequency scale ranging from (1) almost never to (4) almost always. Total scores for state and trait anxiety range from 20 to 80 . Cronbach's alpha of 0.92 (state) and 0.86 (trait) were obtained in the present study.

Depressive symptomatology was measured with the Edinburgh Postnatal Depression Scale (EPDS) validated for use in an antenatal setting [10]. EPDS is a self-administered 10 -item screening questionnaire with each item scored on a 4-point scale (0 to 3) and a total score range of 0-30 [11]. Respondents rate the intensity of depressive symptoms in the preceding 7 days: higher scores indicate more severe depressive symptoms. A cutoff score of $14 / 15$ or more has been found to identify pregnant women with severe depression [10, 12]. However, 11 was selected as the cut-off score, as recommended for high-risk pregnant women [5]. Cronbach's alpha of 0.85 was obtained in the present study.

Basic demographic and medical information included: age, gestational age, parity, previous miscarriages, previous deliveries, complications during previous and present pregnancy and labour, previous infertility problems, marital status, educational level, economic level, and employment status. The educational level was categorized as low (up through elementary school), medium (high school certificate) or high (university degree). The annual income level was categorized as low (9,600-17,999 $€$ or 13.300-25.000 USD), medium (18,000-35,999 $€$ or $25.001-50.170$ USD) or high $(>36,000 €$ or $>$ 50.171USD) [13].

\section{Statistical Analysis}

Statistical analysis was performed using SPSS version 20.0. Descriptive statistics, such as means, standard deviations, and frequencies, were used to represent the demographic characteristics of the participants.
Mean values and standard deviations of the total sum scores of the STAI and EPDS, were also calculated. $\mathrm{P}$ values less than 0.05 were considered significant.

\section{Ethics}

The Research and Ethics Committee of the Elena Benizelou hospital and the General Hospital Alexandra, approved this study protocol. All participants in this study were informed about the scope and the purpose of the study. Eligible women were also assured that the collected data would be used only for the purpose of the study, and that their decision to withdraw would not compromise the standard of the received care. A signed informed consent was obtained from all study participants.

\section{Results}

\section{Characteristics of participants}

The mean age of participants was 32 years. Fiftyfive percent had education beyond high school and $38.3 \%$ had high school and $6.7 \%$ had less than a high school education. Almost $50 \%$ of women participated in the work-force, had low annual family income and the vast majority of women were married. For $61.7 \%$ of the sample this was their first pregnancy and $22.6 \%$ of the women had experienced previous miscarriages. Thirteen percent of the participants had experienced a complication during previous pregnancy or previous labour and specially $11.3 \%$ of participants had experienced a previous preterm birth. The majority of women were hospitalized for premature birth, preterm rapture of membranes, proeclampsia, placenta previa and placenta abruption.

\section{Prevalence of antepartum anxiety and depressive symptoms}

The means for STAI-state and -trait scores were $49.3(S D=11.6)$ and 45.1 (SD=8.8), respectively. 
The mean score for EPDS was 12.5 (SD=6.2). The mean scores of questions of EPDS had a range of (0.21-1.78) with question 4 and 10 to have the maximum and minimum mean score respectively. It is noteworthy, that the $9 \%$ of the participants have answered that quite often or sometimes had the thought of harming themselves. Another $9 \%$ of the participants have answered that hardly ever had the thought of harming themselves. The means of state anxiety, trait anxiety and depression scores in three trimesters of pregnancy are presented in Table 1.

Table 1. Means of state anxiety, trait anxiety and depression scores in three trimesters of pregnancy.

\begin{tabular}{|l|c|c|c|c|}
\hline $\begin{array}{l}\text { Psychological } \\
\text { variables }\end{array}$ & $\begin{array}{c}\text { 1st } \\
\text { trimester }\end{array}$ & $\begin{array}{c}\text { 2nd } \\
\text { trimester }\end{array}$ & $\begin{array}{c}\text { 3rd } \\
\text { trimester }\end{array}$ & $\begin{array}{c}\mathbf{p} \\
\text { value }\end{array}$ \\
\hline $\begin{array}{l}\text { State anxiety, } \\
\text { mean (SD) }\end{array}$ & $46.3(10.8)$ & $50.2(10.2)$ & $53.8(14.8)$ & 0.531 \\
\hline $\begin{array}{l}\text { Trait anxiety, } \\
\text { mean (SD) }\end{array}$ & $47.8(5.1)$ & $43.3(7.7)$ & $45.2(8.5)$ & 0.050 \\
\hline $\begin{array}{l}\text { Depressive } \\
\text { symptomatology, } \\
\text { mean (SD) }\end{array}$ & $15.5(6.8)$ & $12.4(4.8)$ & $16.8(6.7)$ & 0.170 \\
\hline
\end{tabular}

Of the 133 participants assessed $53.4 \%$ had EPDS scores $>11$. Specifically, $50 \%$ of first trimester pregnant women had an EPDS score $>11$. Similarly, $71.4 \%$ of second trimester pregnant women and $80 \%$ of third trimester pregnant women had an EPDS score $>11$. The difference was not significant $(p=0.502)$.

\section{Correlations between anxiety and depressive symptoms}

Statistically significant correlations were found between state anxiety and depressive symptomatology $(r=0.611, p<0.001)$ and between trait anxiety and depressive symptomatology $(r=0.730, p<0.001)$.

\section{Discussion}

According to the authors' knowledge, this is the first study that reports on the incidence of anxiety and depression in a sample of high-risk pregnant women admitted to Greek public hospitals.

The main findings of this study suggest that antenatal depression occurs in almost half of highrisk pregnant women and antenatal anxiety occurs in the majority of high-risk pregnant women. It is extremely interesting that almost one in ten highrisk pregnant women had the thought of harming themselves. In addition, the study findings suggest that high levels of state and trait anxiety were related with high levels of depressive symptomatology. In a previous Greek study conducted at a public hospital was found that the prevalence of antenatal depression in low-risk pregnant women to be about 30\% and the prevalence of antenatal anxiety to be about 45\% [15]. Therefore, it seems that the Greek high-risk pregnant women have significantly higher levels of anxiety and depression than Greek low-risk pregnant women.

The rate of depression is in agreement with previous reports from both European countries $[4,5,7]$. Asian countries [8] and USA [6]. Nevertheless, the rate of anxiety in this study was higher than those reported in study by Thiagayson et al. conducted in Singapore [8]. The high prevalence of anxiety in this study could be attributed to special socio-economic circumstances, such as financial crisis and high rates of unemployment. It is noteworthy, that only $50 \%$ of participants participated in the work force and $50 \%$ of participants had low family annual income. In addition to that, the direct association between poverty and depression is well documented in highincome countries $[16,17]$. Therefore, it could be financial issues may have caused significant worry among Greek pregnant women. 


\section{Conclusion}

According to the findings of this study, about $50 \%$ of pregnant women experience depression symptoms and the vast majority of women experience antenatal anxiety, that not only have deleterious effects on the woman but also on her baby. Midwives and health care professionals who recognise the signs and symptoms of antenatal depression and anxiety can help to identify and prevent them. Incorporating psychosocial interventions in antenatal units or in obstetric clinics that high-risk pregnant women are hospitalized it would facilitate earlier identification and management of antenatal depression and anxiety disorders. In addition, screening tools for identification of antenatal anxiety and depressive symptoms in high-risk pregnancy is crucial.

\section{Acknowledgment}

This study was partly funded by the Institute for Mental Health and Prevention and Therapy of Personality Disorders.

\section{References}

1. Gourounti K, Lykeridou K, Taskou C, Kafetsios K, Sandall J. (2012) A survey of worries of pregnant women: Reliability and validity of the Greek version of the Cambridge Worry Scale. Midwifery, 28(6): 746-753.

2. Grote N, Bridge J, Gavin A, Melville J, lyengar S, Katon W. (2010) A meta-analysis of depression during pregnancy and the risk of preterm birth, low birth weight, and intrauterine growth restriction. Arch Gen Psychiatry, 67: 1012-24.

3. Alder J, Fink N, Bitzer J, et al. (2007) Depression and anxiety during pregnancy: a risk factor for obstetric, fetal and neonatal outcome? A critical review of the literature. Journal of Maternal Fetal Neonatal Medicine, 20(3): 189-209.

4. King N, Chambers J, O'Donnell K, Jayaweera SR, Williamson C, Glover VA. (2010) Anxiety, depression and saliva cortisol in women with a medical disorder during pregnancy. Archive of Womens Mental Health, 13: 339-45

5. Adouard F, Glangeaud-Freudenthal NMC, Golse B. (2005) Validation of the Edinburgh Postnatal Depression Scale (EPDS) in a sample of women with high-risk pregnancies in France. Archive Womens Mental Health, 8: 89-95

6. Brandon A, Trivedi M, Hynan L, Miltenberge P, Labat D, Rifkin J, Stringer A. (2008) Prenatal Depression in Women Hospitalized for Obstetric Risk. National Institute of Health, 69(4): 635-643.

7. Denis A, Michaux P, Callohan S. (2012) Factors implicated in moderating the risk for depression and anxiety in high risk pregnancy. Journal of Reproductive and Infant Psychology, 30(2): 124-134.

8. Thiagayson P, Krishnaswamy G, Lim M, Sung S, Haley C, Fung D, Allen J, Chen H. (2013) Depression and anxiety in Singaporean high-risk pregnancies - prevalence and screening. Gen Hospital Psychiatry, 35(2): 112-116.

9. Spielberger C. Anxiety: Current trends in research. London: Academic Press, 1972.

10. Murray D, Cox J. (1990) Screening for depression during pregnancy with the Edinburgh Postnatal Depression Scale (EPDS). Journal of Reproductive and Infant Psychology, 8: 99107.

11. Cox JL, Holden JM, Sagovsky R. (1987) Detection of postnatal depression: development of the 10 item Edinburgh Postnatal Depression Scale. British Journal Psychiatry, 140: 111-7

12. Murray D, Cox J. (1990) Screening for depression during pregnancy with the Edinburgh Postnatal Depression Scale (EPDS). Journal of Reproductive and Infant Psychology, 8: 99107. 
13. Adewuya AO, Ola BA, Dada AO, Fasoto OO. (2006) Validation of the Edinburgh Postnatal Depression Scale as a screening tool for depression in late pregnancy among Nigerian women. Journal of Psychosomatics in Obstetrics Gynecology, 2: 267-72

14. Hellenic Statistical Authority. 2006, Annual income for Greek population report. Retrieved January 15, 2007, from http:// www.statistics.gr/portal/page/portal/ver- 1/ESYE/BUCKET/ A0103/Other/A0103_SJO46_TB_4Y_00_2006_03_F_GR.pdf

15. Gourounti K., Anagnostopoulos F., Lykeridou K., Griva F., Vaslamatzis G. (2013) Prevalence of women's worries, anxiety and depression during pregnancy in a public hospital setting in Greece. Journal of Clinical and Expimental Obstetrics Gynecology, 40(4): 581-583.

16. Leigh B, Milgrom J. (2008) Risk factors for antenatal depression, postnatal depression and parenting stress. BMC Psychiatry, 8: 24.

17. Bodecs $T$, Horvath $B$, Kovacs $L$, Diffelne Nemeth $M$, Sandor J. (2009) Prevalence of depression and anxiety on a population based Hungarian sample. Orv Hetil, 150(41): 1888-1893.

\section{Comment on this article:}

\section{$9[8$ in $8+\mathbf{S}$ P}

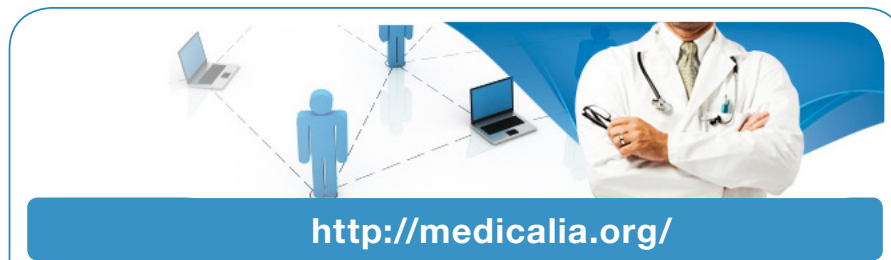

Where Doctors exchange clinical experiences, review their cases and share clinical knowledge. You can also access lots of medical publications for free. Join Now!

\section{Publish with iMedPub \\ http://www.imed.pub}

International Archives of Medicine is an open access journal publishing articles encompassing all aspects of medical science and clinical practice. IAM is considered a megajournal with independent sections on all areas of medicine. IAM is a really international journal with authors and board members from all around the world. The journal is widely indexed and classified Q1 in category Medicine. 